\title{
ON THE PRIME SPECTRUM OF MODULES
}

\author{
H. ANSARI-TOROGHY AND S. S. POURMORTAZAVI
}

Received 18 January, 2014

\begin{abstract}
Let $R$ be a commutative ring and let $M$ be an $R$-module. Let us denote the set of all prime submodules of $M$ by $\operatorname{Spec}(M)$. In this article, we explore more properties of strongly top modules and investigate some conditions under which $\operatorname{Spec}(M)$ is a spectral space.
\end{abstract}

2010 Mathematics Subject Classification: 13C13; 13C99

Keywords: prime spectrum, Zariski topology, spectral space

\section{INTRODUCTION, ETC}

Throughout this article, all rings are commutative with identity elements, and all modules are unital left modules. $\mathbb{N}, \mathbb{Z}$, and $\mathbb{Q}$ will denote respectively the natural numbers, the ring of integers and the field of quotients of $\mathbb{Z}$. If $N$ is a subset of an $R$-module $M$, then $N \leq M$ denotes $N$ is an submodule of $M$.

Let $M$ be an $R$-module. For any submodule $N$ of $M$, we denote the annihilator of $M / N$ by $(N: M)$, i.e. $(N: M)=\{r \in R \mid r M \subseteq N\}$. A submodule $P$ of $M$ is called prime if $P \neq M$ and whenever $r \in R$ and $e \in M$ satisfy $r e \in P$, then $r \in(P: M)$ or $e \in P$.

The set of all prime submodule of $M$ is denoted by $\operatorname{Spec}(M)$ (or $X$ ). For any ideal $I$ of $R$ containing $A n n(M), \bar{I}$ and $\bar{R}$ will denote $I / A n n(M)$ and $R / A n n(M)$, respectively. Also the map $\psi: \operatorname{Spec}(M) \rightarrow \operatorname{Spec}(\bar{R})$ given by $P \mapsto \overline{(P: M)}$ is called the natural map of $X . M$ is called primeful (resp. $X$-injective) if either $M=\mathbf{0}$ or $M \neq \mathbf{0}$ and the natural map $\psi$ is surjective (resp. if either $X=\varnothing$ or $X \neq \varnothing$ and natural map $\psi$ is injective). (See [3,11] and [13].)

The Zariski topology on $X$ is the topology $\tau$ described by taking the set $\Omega=$ $\{V(N) \mid N$ is a submodule of $M\}$ as the set of closed sets of $X$, where $V(N)=\{P \in$ $X \mid(P: M) \supseteq(N: M)\}[11]$.

The quasi-Zariski topology on $X$ is described as follows: put $V^{*}(N)=\{P \in$ $X \mid P \supseteq N\}$ and $\Omega^{*}=\left\{V^{*}(N) \mid N\right.$ is a submodule of $\left.M\right\}$. Then there exists a topo$\operatorname{logy} \tau^{*}$ on $X$ having $\Omega^{*}$ as the set of it's closed subsets if and only if $\Omega^{*}$ is closed under the finite union. When this is the case, $\tau^{*}$ is called a quasi-Zariski topology on $X$ and $M$ is called a top $R$-module [15]. 
Let $Y$ be a topological space. $Y$ is irreducible if $Y \neq \varnothing$ and for every decomposition $Y=A_{1} \cup A_{2}$ with closed subsets $A_{i} \subseteq Y, i=1,2$, we have $A_{1}=Y$ or $A_{2}=Y$. A subset $T$ of $Y$ is irreducible if $T$ is irreducible as a space with the relative topology. For this to be so, it is necessary and sufficient that, for every pair of sets $F, G$ which are closed in $Y$ and satisfy $T \subseteq F \cup G, T \subseteq F$ or $T \subseteq G$. Let $F$ be a closed subset of $Y$. An element $y \in Y$ is called a generic point of $Y$ if $Y=\operatorname{cl}(\{y\})$ (here for a subset $Z$ of $Y, \operatorname{cl}(Z)$ denotes the topological closure of $Z$ ).

A topological space $X$ is a spectral space if $X$ is homeomorphic to $\operatorname{Spec}(S)$ with the Zariski topology for some ring $S$. This concept plays an important role in studying of algebraic properties of an $R$-module $M$ when we a have a related topology. For an example, when $\operatorname{Spec}(M)$ is homeomorphic to $\operatorname{Spec}(S)$, where $S$ is a commutative ring, we can transfer some of known topological properties of $\operatorname{Spec}(S)$ to $\operatorname{Spec}(M)$ and then by using these properties explore some of algebraic properties of $M$.

Spectral spaces have been characterized by $\mathrm{M}$. Hochster as quasi-compact $T_{0^{-}}$ spaces $X$ having a quasi-compact open base closed under finite intersection and each irreducible closed subset of $X$ has a generic point [9, p. 52, Proposition 4].

The concept of strongly top modules was introduced in [2] and some of its properties have been studied. In this article, we get more information about this class of modules and explore some conditions under which $\operatorname{Spec}(M)$ is a spectral space for its Zariski or quasi-Zariski topology.

In the rest of this article, $X$ will denote $\operatorname{Spec}(M)$. Also the set of all maximal submodules of $M$ is denoted by $\operatorname{Max}(M)$.

\section{MAIN RESULTS}

Definition 1 (Definition 3.1 in [1]). Let $M$ be an $R$-module. $M$ is called a strongly top module if for every submodule $N$ of $M$ there exists an ideal $I$ of $R$ such that $V^{*}(N)=V^{*}(I M)$.

Definition 2 (Definition 3.1 in [2]). Let $M$ be an $R$-module. $M$ is called a strongly top module if $M$ is a top module and $\tau^{*}=\tau$.

Remark 1. Definition 1 and Definition 2 are equivalent. This follows from the fact that if $N$ is a submodule of $M$, then by [11, Result 3], we have

$$
V(N)=V((N: M) M)=V^{*}((N: M) M) .
$$

Remark 2 (Theorem 6.1 in [11]). Let $M$ be an $R$-module. Then the following are equivalent:

(a) $(X, \tau)$ is a $T_{0}$ space;

(b) The natural map of $X$ is injective;

(c) $V(P)=V(Q)$, that is, $(P: M)=(Q: M)$ implies that $P=Q$ for any $P, Q \in X$ 
(d) $\left|\operatorname{Spec}_{p}(M)\right| \leq 1$ for every $p \in \operatorname{Spec}(R)$.

Remark 3. $\quad$ (a) Let $M$ be an $R$-module and $p \in \operatorname{Spec}(R)$. The saturation of a submodule $N$ with respect to $p$ is the contraction of $N_{p}$ in $M$ and denoted by $S_{p}(N)$. It is known that

$$
S_{p}(N)=N^{e c}=\{x \in M \mid t x \in N \text { for some } t \in R \backslash p\} .
$$

(b) Let $M$ be an $R$-module and $N \leq M$. The radical of $N$, denoted by $\operatorname{rad}(N)$, is the intersection of all prime submodules of $M$ containing $N$; that is, $\operatorname{rad}(N)=\bigcap_{P \in V^{*}(N)} P([14])$.

(c) A topological space $X$ is Noetherian provided that the open (respectively, closed) subsets of $X$ satisfy the ascending (respectively, descending) chain condition ([4, p. 79, Exercises 5-12]).

Proposition 1. Let $M$ be an strongly top module and $\psi$ be the natural map of $X$. Then

(a) $(X, \tau)=\left(X, \tau^{*}\right) \cong \operatorname{Im} \psi$.

(b) If $X$ is Noetherian, then $X$ is a spectral space.

Proof. (a) By [15, Theorem 3.5] and Remark 2, $\left.\psi\right|_{I m \psi}$ is bijective. Also we have

$$
\psi(V(N))=\{\overline{(P: M)} \mid P \in X,(P: M) \supseteq(N: M)\} .
$$

Now by [11, Proposition 3.1] and the above arguments, $\psi$ is continuous and a closed map. Consequently we have $(X, \tau)=\left(X, \tau^{*}\right) \cong \operatorname{Im} \psi$.

(b) Let $Y=V^{*}(N)$ be an irreducible closed subset of $X$. Now by [6, Theorem 3.4], we have

$$
V^{*}(N)=V^{*}(\operatorname{rad}(N))=c l(\{\operatorname{rad}(N)\}) .
$$

Hence $Y$ has a generic point. Also $X$ is Noetherian and it is a $T_{0}$-space by [6, Proposition 3.8 (i)]. Hence it is a spectral space by [9, Pages 57 and 58].

An $R$ - module $M$ is said to be a weak multiplication module if either $X=$ $\operatorname{Spec}(M)=\varnothing$ or $X \neq \varnothing$ and for every prime submodule $P$ of $M$, we have $P=I M$ for some ideal $I$ of $R$ (see [5]).

The following theorem extends [1, Proposition 3.5], [1, Corollary 3.6], [1, Theorem 3.9 (1)], and [1, Theorem 3.9 (7)]. In fact, in part (a) of this theorem, we withdraw the restrictions of finiteness and Noetherian property from [1, Proposition 3.5] and [1, Corollary 3.6], respectively. In part (b), we remove the conditions " $M$ is primeful " and " $R$ is a Noetherian ring " in [1, Theorem $3.9(1)]$ and instead of them, we put the weaker conditions " $\operatorname{Im}(\psi)$ is closed in $\operatorname{Spec}(\bar{R})$ " and " $\operatorname{Spec}(\bar{R})$ is a Noetherian space ". In part (c), we withdraw the condition " $R$ has Noetherian spectrum " from [1, Theorem 3.9 (7)] and put the weaker condition " the intersection of every infinite family of maximal ideals of $R$ is zero".

Theorem 1. Let $M$ be an R-module. Then we have the following. 
(a) Let $\left(M_{i}\right)_{i \in I}$ be a family of $R$-modules and let $M=\oplus_{i \in I} M_{i}$. If $M$ is an strongly top $R$-module, then each $M_{i}$ is an strongly top $R$-module.

(b) If $M$ be an strongly top $R$-module and $\psi$ be the natural map of $X$, then we have

(i) If $\operatorname{Im}(\psi)$ is closed in $\operatorname{Spec}(\bar{R})$, then $(X, \tau)=\left(X, \tau^{*}\right)$ is a spectral space.

(ii) If Spec $(\bar{R})$ is Noetherian, then $(X, \tau)=\left(X, \tau^{*}\right)$ is a spectral space.

(c) Suppose $R$ is a one dimensional integral domain such that the intersection of every infinite family of its maximal ideals is zero. If $M$ is a weak multiplication $R$-module, then $M$ is a top module.

Proof. (a) Each $M_{i}$ is a homomorphic image of $M$, hence it is strongly top by [1, Proposition 3.3].

(b) (i) By Proposition 1, we have $(X, \tau)=\left(X, \tau^{*}\right) \cong \operatorname{Im}(\psi)$. Now the claim follows by [11, Theorem 6.7].

(ii) As $\operatorname{Spec}(\bar{R})$ is Noetherian, $\operatorname{Im}(\psi)$ is also Noetherian. Now the claim follows from Proposition 1.

(c) Use the technique of [3, Theorem 3.18].

The following theorem extends [1, Theorem 3.9(3) ].

Theorem 2. Suppose $R$ is a one dimensional integral domain such that the intersection of every infinite family of its maximal ideals is zero. If $M$ is $X$-injective with $S_{0}(\mathbf{0}) \subseteq \operatorname{rad}(\mathbf{0})$, then $M$ is a top module.

Proof. If $S_{0}(\mathbf{0})=M$, then $X=\varnothing$ and there is nothing to prove. Otherwise, by [12, Corollary 3.7], $S_{0}(\mathbf{0})$ is a prime submodule so that $S_{0}(\mathbf{0})=\operatorname{rad}(\mathbf{0})$. Hence the natural map $f: \operatorname{Spec}\left(M / S_{0}(\mathbf{0})\right) \rightarrow \operatorname{Spec}(M)$ is a homeomorphism by [7, Proposition 1.4]. But by [3, Theorem 3.7 (a)] and [3, Theorem 3.15 (e)], $M / S_{0}(\mathbf{0})$ is a weak multiplication module. Now the result follows because by Theorem 1 (c), $M / S_{0}(\mathbf{0})$ is a top module.

Let $M$ be an $R$-module. Then $M$ is called a content module if for every $x \in M$, $x \in c(x) M$, where $c(x)=\bigcap\{I \mid I$ is an ideal of $R$ such that $x \in I M\}$ (see [13, p. 140]).

In below we generalize [1, Theorem 3.9(4)].

Theorem 3. Suppose $R$ is a one dimensional integral domain and let $M$ be a content $R$-module. Then we have the following.

(a) If $M$ is $X$-injective, then $M$ is a top module.

(b) If $M$ is $X$-injective and $S_{0}(\boldsymbol{0}) \subseteq \operatorname{rad}(\boldsymbol{0})$, then $M$ is an strongly top module. Furthermore, if Spec $(\bar{R})$ is Noetherian, then $\left(X, \tau^{*}\right)$ is spectral. 
Proof. (a) By [3, Theorem 3.21], we have

$\operatorname{Spec}(M)=\left\{S_{p}(p M) \mid p \in V(\operatorname{Ann}(M)), S_{p}(p M) \neq M\right\}=\left\{S_{0}(\mathbf{0})\right\} \cup \operatorname{Max}(M)$,

where

$$
\operatorname{Max}(M)=\{p M \mid p \in \operatorname{Max}(R), p M \neq M\} .
$$

Let $N \leq M$ and let $N \nsubseteq S_{0}(\mathbf{0})$. Then

$$
\operatorname{rad}(N)=\bigcap_{N \subseteq P \in \operatorname{Spec}(M)} P=\bigcap_{N \subseteq P \in \operatorname{Max}(M)} P .
$$

So by the above arguments, there is an index set $I$ such that $\operatorname{rad}(N)=\bigcap_{i \in I}\left(p_{i} M\right)$. Since $M$ is content module,

$$
V^{*}(N)=V^{*}(\operatorname{rad}(N))=V^{*}\left(\bigcap_{i \in I}\left(p_{i} M\right)\right)=V\left(\left(\bigcap_{i \in I} p_{i} M\right) .\right.
$$

Now if $N \subseteq S_{0}(\mathbf{0})$, then by [10, Lemma 2],

$$
\begin{aligned}
V^{*}(N) & =V^{*}(\operatorname{rad}(N))=V^{*}\left(S_{0}(\mathbf{0}) \cap\left(\bigcap_{i \in I}\left(p_{i} M\right)\right)\right) \\
& =V^{*}\left(S_{0}(\mathbf{0}) \cap\left(\left(\bigcap_{i \in I} p_{i}\right) M\right)\right) \\
& =V^{*}\left(S_{0}(\mathbf{0})\right) \cup V^{*}\left(\left(\bigcap_{i \in I} p_{i}\right) M\right) \\
& =V^{*}\left(S_{0}(\mathbf{0})\right) \cup V\left(\left(\bigcap_{i \in I} p_{i}\right) M\right) .
\end{aligned}
$$

By the above arguments, it follows that $M$ is a top module.

(b) By [3, Theorem 3.21],

$\operatorname{Spec}(M)=\left\{S_{0}(\mathbf{0})\right\} \cup \operatorname{Max}(M)$ and $\operatorname{Max}(M)=\{p M \mid p \in \operatorname{Max}(R), p M \neq M\}$.

Let $N \leq M$. If $N \subseteq S_{0}(\mathbf{0})$, then $V^{*}(N)=V^{*}(\mathbf{0})=X$. Otherwise, we have $\operatorname{rad}(N)=\bigcap_{i \in I}\left(p_{i} M\right)$ by [3, Theorem 3.21]. Since $M$ is content, by [11, Result 3] we have

$$
V^{*}(N)=V^{*}(\operatorname{rad}(N))=V^{*}\left(\bigcap_{i \in I}\left(p_{i} M\right)\right)=V\left(\left(\bigcap_{i \in I} p_{i}\right) M\right) .
$$

Hence $M$ is an strongly top module. The second assertion follows from Theorem 1 (b).

Theorem 4. If $M$ is content weak multiplication, then $M$ is an strongly top module. Moreover, if $\operatorname{Spec}(R)$ is Noetherian, then $\left(X, \tau^{*}\right)$ is a spectral space. 
Proof. Let $N \leq M$. Then we have

$$
V^{*}(N)=V^{*}(\operatorname{rad}(N))=V^{*}\left(\bigcap_{N \leq P} P\right) .
$$

Since $M$ is a weak multiplication module, for each prime submodule $P$ of $M$ containing $N$, there exists an ideal $I_{P}$ of $R$ such that $P=I_{P} M$. Hence since $M$ is a content module,

$$
V^{*}(N)=V^{*}\left(\bigcap_{N \leq P}\left(I_{P} M\right)\right)=V^{*}\left(\left(\bigcap_{N \leq P} I_{P}\right) M\right) .
$$

This implies that $M$ is an strongly top module. Since $\operatorname{Spec}(R)$ is Noetherian, so is $\operatorname{Spec}(\bar{R})$. Hence by Theorem $1(\mathrm{~b}),\left(X, \tau^{*}\right)$ is a spectral space.

Theorem 5. Let $R$ be a one-dimensional integral domain and let $M$ be an $X$ injective $R$-module such that $S_{0}(\mathbf{0}) \subseteq \operatorname{rad}(\mathbf{0})$. If the intersection of every infinite number of maximal submodules of $M$ is zero, then $M$ is strongly top and $\left(X, \tau^{*}\right)$ is a spectral space.

Proof. If $S_{0}(\mathbf{0})=M$, then $X=\varnothing$ and there is nothing to prove. Otherwise, by [3, Theorem 3.21], we have $\operatorname{Spec}(M)=\left\{S_{0}(\mathbf{0})\right\} \cup \operatorname{Max}(M)$ and $\operatorname{Max}(M)=$ $\{p M \mid p \in \operatorname{Max}(R), p M \neq M\}$. Now let $N \leq M$. If $N=\mathbf{0}$, then claim clear because $V^{*}(N)=V^{*}(\mathbf{0})=V^{*}(0 M)=\operatorname{Spec}(M)$. So we assume that $N \neq \mathbf{0}$. We consider two cases.

(1) $N \subseteq S_{0}(\mathbf{0})$. In this case, we have $V^{*}(N)=V^{*}(\mathbf{0})=V^{*}(0 M)=\operatorname{Spec}(M)$.

(2) $N \nsubseteq S_{0}(\mathbf{0})$. Then since $N \neq 0$ and the intersection of every infinite number of maximal submodules of $M$ is zero, $\operatorname{rad}(N)=\bigcap_{i=1}^{n}\left(p_{i} M\right)$, where $p_{i} M \in$ $\operatorname{Max}(M)$ for each $i(1 \leq i \leq n)$. Hence we have

$$
V^{*}(N)=V^{*}(\operatorname{rad}(N))=V^{*}\left(\bigcap_{i=1}^{n}\left(p_{i} M\right)\right) .
$$

Now we show that $V^{*}\left(\bigcap_{i=1}^{n}\left(p_{i} M\right)\right)=V^{*}\left(\left(\bigcap_{i=1}^{n} p_{i}\right) M\right)$. Clearly, $V^{*}\left(\bigcap_{i=1}^{n}\left(p_{i} M\right)\right) \subseteq V^{*}\left(\left(\bigcap_{i=1}^{n} p_{i}\right) M\right)$. Too see this reverse inclusion, let $P \in V^{*}\left(\left(\bigcap_{i=1}^{n} p_{i}\right) M\right)$. If $P=S_{0}(\mathbf{0})$, then $\left(\bigcap_{i=1}^{n} p_{i}\right) M \subseteq S_{0}(\mathbf{0})$ implies that $\bigcap_{i=1}^{n} p_{i} \subseteq\left(\left(\bigcap_{i=1}^{n} p_{i}\right) M: M\right) \subseteq\left(S_{0}(\mathbf{0}): M\right)=0$. Thus, there exists $j(1 \leq j \leq$ $n$ ) such that $p_{j}=0$, a contradiction. Hence we must have $P=q M$, where $q \in$ $\operatorname{Max}(R)$. Then, similar the above arguments, there exists $j(1 \leq j \leq n)$ such that $q=p_{j}$. Therefore, $P=q M=p_{j} M \in V^{*}\left(\bigcap_{i=1}^{n}\left(p_{i} M\right)\right)$. So we have

$$
V^{*}(N)=V^{*}\left(\bigcap_{i=1}^{n}\left(p_{i} M\right)\right)=V^{*}\left(\left(\bigcap_{i=1}^{n} p_{i}\right) M\right) .
$$

Hence $M$ is strongly top so that $\tau=\tau^{*}$. On the other hand, $\tau=\tau^{*}$ is a subset of a finite complement topology. This implies that $\left(X, \tau^{*}\right)$ is Noetherian. Now by Proposition $1,\left(X, \tau^{*}\right)=(X, \tau)$ is spectral. 
Theorem 6. If for each submodule $N$ of $M, \operatorname{rad}(N)=\sqrt{(N: M)} M$, then $M$ is an strongly top module. Moreover, if $\operatorname{Spec}(R)$ is Noetherian, then $\left(X, \tau^{*}\right)$ is spectral.

Proof. Let $N \leq M$. Then we have

$$
\begin{aligned}
V^{*}(N)=V^{*}(\operatorname{rad}(N)) & =V^{*}(\sqrt{(N: M)} M) \\
& =V(\sqrt{(N: M)} M)=V(\operatorname{rad}(N))=V(N) .
\end{aligned}
$$

Hence $M$ is an strongly top module. Now the result follows by using similar arguments as in the proof of Theorem 4.

Remark 4. Theorems 4, 5, and 6 improve respectively [1, Theorem 3.9(5)], [1, Theorem 3.9(8)], and [1, Theorem 3.9(6)]. They show that the notion of "top modules " can be replaced by "strongly top modules" and the proofs can be shortened considerably.

In below we generalize [1, Theorem 3.36].

Theorem 7. Let $M$ be a primeful R-module. Then we have the following.

(a) If $(X, \tau)$ is discrete, then $\operatorname{Spec}(M)=\operatorname{Max}(M)$.

(b) If $R$ is Noetherian and $\operatorname{Spec}(M)=\operatorname{Max}(M)$, then $(X, \tau)$ is a finite discrete space.

Proof. (a) Since $(X, \tau)$ is discrete, it is a $T_{1}$-space. Now by [3, Theorem 4.3], we have $\operatorname{Spec}(M)=\operatorname{Max}(M)$.

(b) By [3, Theorem 4.3], $\operatorname{Spec}(\bar{R})=\operatorname{Max}(\bar{R})$. Hence $\bar{R}$ is Artinian. Now by [3, Theorem 4.3], $(X, \tau)$ is a $T_{0}$-space. Thus by Remark $2, M$ is $X$-injective. But $M$ is a cyclic $\bar{R}$-module and hence a cyclic $R$-module by [3, Remark 3.13] and [3, Theorem 3.15]. Also $(\operatorname{Spec}(M), \tau)$ is homoeomorphic to $\operatorname{Spec}(\bar{R})$ by [11, Theorem 6.5(5)]. Hence $X$ is a finite discrete space by [4, Chapter 8, Exe 2].

It is well known that if $R$ is a PID and $\operatorname{Max}(R)$ is not finite, then the intersection every infinite number of maximal ideals of $R$ is zero. Now it is natural to ask the following question: Is the same true when $R$ is a one dimensional integral domain with infinite maximal ideals? In below, we show that this true when $\operatorname{Spec}(R)$ is a Noetherian space. Although this is not a simple fact, it used by some authors without giving any proof.

Theorem 8. (a) Let $I$ be an ideal of $R$ and let $k, n \in \mathbb{N}$. Then $\left(\sqrt{I}: a^{k}\right)=$ $\left(\sqrt{I}: a^{n}\right)$.

(b) Let $I$ be an ideal of $R$ and let $a \in R, n \in \mathbb{N}$. Then $\sqrt{I}=\sqrt{\left(\sqrt{I}: a^{n}\right)} \cap$ $\sqrt{\left\langle\sqrt{I}, a^{n}\right\rangle}$. 
(c) Suppose Spec $(R)$ is a Noetherian topological space. Then for every ideal I of $R, \sqrt{I}$ has a primary decomposition.

(d) Suppose $R$ is a one dimensional integral domain and $\operatorname{Spec}(R)$ is a Noetherian topological space. Then the intersection of every infinite number of maximal ideals is zero.

Proof. (a) It is clear.

(b) Let $f \in \sqrt{\left(\sqrt{I}: a^{n}\right)} \cap \sqrt{\left\langle\sqrt{I}, a^{n}\right\rangle}$. Then there is $m \in \mathbb{N}$ such that $f^{m} \in(\sqrt{I}$ : $\left.a^{n}\right) \cap\left\langle\sqrt{I}, a^{n}\right\rangle$. It follows that $f^{m}=g+x a^{n}$ for some $g \in \sqrt{I}$ and $x \in R$ and we also get $a^{n} f^{m} \in \sqrt{I}$. Hence $a^{n} f^{m}=a^{n} g+x a^{2 n}$. This implies that $x a^{2 n} \in \sqrt{I}$ and so $x \in\left(\sqrt{I}: a^{n}\right)$ by part (a). Thus $x a^{n} \in \sqrt{I}$. It follows that $f \in \sqrt{I}$. The reverse inclusion is clear.

(c) Set $\Sigma=$

$\{\sqrt{I} \mid I$ is a proper ideals of $R$ and $\sqrt{I}$ doesn't have any primary decomposition $\}$.

Since $\operatorname{Spec}(R)$ is Noetherian, the radicals of ideals satisfy the a.c.c. condition. So $\Sigma$ has a maximal member, $\sqrt{I_{0}}$ say. Thus $\sqrt{I_{0}} \notin \operatorname{Spec}(R)$. In other words,

$$
\exists a, b \in R \text { s.t. } a b \in \sqrt{I_{0}} \text { and } a \notin \sqrt{I_{0}} \text { and } b \notin \sqrt{I_{0}} \text {. }
$$

By part (b) we have $\sqrt{I_{0}}=\sqrt{\left(\sqrt{I_{0}}: b\right)} \cap \sqrt{\left\langle\sqrt{I_{0}}, b\right\rangle}$. Further, $\sqrt{I_{0}} \subsetneq \sqrt{\left(\sqrt{I_{0}}: b\right)}$ and $\sqrt{I_{0}} \subsetneq \sqrt{\left\langle\sqrt{I_{0}}, b\right\rangle}$. Since $\sqrt{\left(\sqrt{I_{0}}: b\right)}$ and $\sqrt{\left\langle\sqrt{I_{0}}, b\right\rangle}$ have primary decompositions by hypothesis, $\sqrt{I_{0}}$ has a primary decomposition, a contradiction.

(d) Since $R$ is one dimensional integral domain, $\operatorname{Spec}(R)=\{0\} \cup \operatorname{Max}(R)$. Suppose $\left\{m_{i}\right\}_{i \in I}$ is an infinite family of maximal ideals of $R$ such that $\bigcap_{i \in I} m_{i} \neq 0$. By part (c), $\sqrt{\bigcap_{i \in I} m_{i}}$ has a primary decomposition. Hence

$$
\sqrt{\bigcap_{i \in I} m_{i}}=\bigcap_{j=1}^{n} m_{j}^{\prime}, \quad m_{j}^{\prime} \in \operatorname{Max}(R) .
$$

This implies that $\left\{m_{i}\right\}_{i \in I}$ is a finite family, a contradiction. So the proof is compeleted.

Example 1 . We show that $\mathbb{Z}[i \sqrt{5}]$ is a one dimensional Noetherian integral domain which has infinite number of maximal ideals and it is not a PID. To see this, let $\phi: \mathbb{Z}[X] \rightarrow \mathbb{Z}[i \sqrt{5}]$ be the natural epimorphism given by $p(x) \mapsto p(i \sqrt{5})$. by using [8] or [16], one can see that

$\operatorname{Spec}(\mathbb{Z}[X])=\{\langle p\rangle,\langle f\rangle,\langle q, g\rangle \mid p$ and $q$ are prime numbers, $f$ is a primary irreducible polynomial in $\mathbb{Q}[X]$, and $g$ is an irreducible polynomial in $\left.\mathbb{Z}_{q}[X]\right\}$.

Now we have $\operatorname{ker} \phi=\left\langle X^{2}+5\right\rangle$. A simple verification shows that

$$
\operatorname{Spec}(\mathbb{Z}[i \sqrt{5}])=\{0\} \cup \operatorname{Max}(\mathbb{Z}[i \sqrt{5}])
$$




$$
=\{0\} \cup\left\{\langle q, g(\sqrt{-5})\rangle \mid\langle q, g\rangle \in \operatorname{Spec}(\mathbb{Z}[X]) \text { and } X^{2}+5 \in\langle q, g\rangle\right\} .
$$

Further $\mathbb{Z}[i \sqrt{5}]$ contains a finite number elements which are invertible by [17, $\mathrm{p}$. $38]$. So $\mathbb{Z}[i \sqrt{5}]$ is a Noetherian one dimensional integral domain with infinite number of maximal ideals. Hence the intersection of every infinite number of maximal ideals of $\mathbb{Z}[i \sqrt{5}]$ is zero by Theorem 8 (c). Note that $\mathbb{Z}[i \sqrt{5}]$ is not a PID by [17, p. 38].

\section{ACKNOWLEDGEMENT}

The authors would like to thank the referee for the careful reading of our manuscript and valuable comments.

\section{REFERENCES}

[1] A. Abbasi and D. Hassanzadeh-Lelekaami, "Modules and spectral spaces," Comm. Algebra, vol. 40, no. 11, pp. 4111-4129, 2012, doi: 10.1080/00927872.2011.602273.

[2] H. Ansari-Toroghy and S. Keyvani, "Strongly top modules," Bull. Malaysian. Math. Sci. Soc.(2), vol. 37, no. 1, pp. 73-82, 2014.

[3] H. Ansari-Toroghy and R. Ovlyaee-Sarmazdeh, "On the prime spectrum of X-injective modules," Comm. Algebra, vol. 38, no. 7, pp. 2606-2621, 2010, doi: 10.1080/00927870903036339.

[4] M. F. Atiyah and I. G. Macdonald, Introduction to commutative algebra. Addison-Wesley Reading, 1969 , vol. 2

[5] A. Azizi, "Weak multiplication modules," Czechoslovak Math. J., vol. 53, no. 3, pp. 529-534, 2003, doi: 10.1023/B:CMAJ.0000024500.35257.39.

[6] M. Behboodi and M. Haddadi, "Classical Zariski topology of modules and spectral spaces I," International Electronic Journal of Algebra, vol. 4, pp. 104-130, 2008.

[7] M. Behboodi and M. Haddadi, "Classical Zariski topology of modules and spectral spaces II," International Electronic Journal of Algebra, vol. 4, pp. 131-148, 2008.

[8] D. Eisenbud and J. Harris, The Geometry of Schemes. New York, Inc: Springer-Verlag, 2000.

[9] M. Hochster, "Prime ideal structure in commutative rings," Trans. Amer. Math. Soc., vol. 142, pp. 43-60, 1969, doi: 10.1090/S0002-9947-1969-0251026-X.

[10] C. P. Lu, "M-radicals of submodules in modules," Math. Japon., vol. 34, no. 2, pp. 211-219, 1989.

[11] C. P. Lu, "The Zariski topology on the prime spectrum of a module," Houston J. Math., vol. 25, no. 3, pp. 417-432, 1999.

[12] C. P. Lu, "Saturations of submodules," Comm. Algebra, vol. 31, no. 6, pp. 2655-2673, 2003, doi: 10.1081/AGB-120021886.

[13] C. P. Lu, "A module whose prime spectrum has the surjective natural map," Houston J. Math., vol. 33, no. 1, pp. 125-143, 2007.

[14] R. McCasland and M. Moore, "On radicals of submodules," Comm. Algebra, vol. 19, no. 5, pp. 1327-1341, 1991, doi: 10.1080/00927879108824205.

[15] R. McCasland, M. Moore, and P. Smith, "On the spectrum of a module over a commutative ring," Comm. Algebra, vol. 25, no. 1, pp. 79-103, 1997, doi: 10.1080/00927879708825840.

[16] D. Mumford, The Red Book of Varieties and Schemes. Springer-Verlag, 1980.

[17] D. Sharpe, Rings and factorization. CUP Archive, 1987. 
Authors' addresses

H. Ansari-Toroghy

University of Guilan, Department of pure Mathematics, Faculty of mathematical Sciences, P. O. Box 41335-19141, Rasht, Iran.

E-mail address: ansari@guilan.ac.ir

\section{S. S. Pourmortazavi}

University of Guilan, Department of pure Mathematics, Faculty of mathematical Sciences, P. O. Box 41335-19141, Rasht, Iran.

E-mail address: mortazaviephd.guilan.ac.ir 